\title{
Discrete states in light-like linear dilaton background
}

\author{
Pei-Ming Ho and Sheng-Yu Darren Shih \\ Department of Physics and Center for Theoretical Sciences, \\ National Taiwan University, Taipei 10617, Taiwan, R.O.C. \\ E-mail: pmho@phys.ntu.edu.tw, darrenj_shih@yahoo.com
}

\begin{abstract}
We study the spectrum of bosonic strings in the light-like linear dilaton background and find discrete states. These are physical states which exist only at specific values of momentum. All discrete states except one generate spacetime symmetries. The exceptional discrete state corresponds to constraints which are deformations of conservation laws. The constraints resemble those arising from symmetries, and are equally powerful, suggesting that our notion of symmetry should be generalized.
\end{abstract}

Keywords: Conformal Field Models in String Theory, Bosonic Strings. 


\section{Contents}

1. Introduction 1

2. Discrete states 3

2.1 Discrete states in 2D with space-like linear dilaton background 3

2.2 Discrete states in 26D flat space

3. Discrete states in light-like linear dilaton background 5

3.1 Discrete states at massless level 6

3.2 No-ghost theorem 8

4. Scattering amplitude in light-like linear dilaton background 10

4.1 Three-point functions 11

4.2 Four-point functions

4.2.1 Two tachyons and two massless vectors 13

4.3 Summary of correlation functions 14

5. Field theory model 15

6. Concluding remarks 17

A. Path integral for the linear dilaton background 19

\section{Introduction}

The original motivation of this work is to understand string theory in time-dependent backgrounds. Our strategy is to start with the simplest example: bosonic string theory in 26 dimensions with a light-like linear dilaton background. This is an extremely simple case for which the worldsheet conformal field theory can be found in textbooks [1]. In many aspects, backgrounds depending on a light-cone coordinate are much easier than generic time-dependent backgrounds. Hence there are much recent interest in backgrounds with light-like dependence [2-5], taking aim at applications to cosmology. In particular, the matrix model of type II A string theory in the light-like linear dilaton background was proposed in [2]. Space-like (e.g. $c=1$ noncritical string [6]) and time-like [7] linear dilaton backgrounds have also been considered before.

This work is focused on a very simple but intriguing property of the light-like linear dilaton background that has never been emphasized before to the best of our knowledge. Recall that in the flat background there are 26 spacetime translation symmetries generated 
by 26 physical states $\alpha_{-1}^{\mu}|0, k=0\rangle$ (for an open string or half of a closed string) corresponding to the vertex operators $\partial X^{\mu}$. We will refer to these states as "discrete states" for reasons that will be explained below. Remarkably, although the translation symmetry in a light-cone coordinate is broken after turning on the dilaton background, we still find 26 (physical) discrete states, including 25 generators of translation symmetry. The additional discrete state which does not correspond to a translation symmetry, and its implications, will be the focus of this paper.

In the next section, we briefly review discrete states in the $2 \mathrm{D}$ string theory with a space-like linear dilaton background, and extend its definition to other backgrounds in string theory. These states are physical states (i.e., they satisfy Virasoro constraints) in the old covariant quantization. They are not spurious states, and their norms can be positive, zero or even negative. Their existence signals a loop-hole in the no-ghost theorem which claims the equivalence between the light-cone gauge and old covariant quantization. The loop-hole is not fatal, and the perturbation theory is still unitary due to the discrete nature of these states. More importantly, we believe that they play a special role in string theory, that is, what we see in 2D string theory is not exceptional. They correspond to spacetime symmetries not only in 2D string theory but also in the 26D string theory in flat spacetime.

In section 3, we find discrete states at the massless level also in the 26D bosonic string theory in the light-like linear dilaton background. We extend the no-ghost theorem for flat spacetime to the linear dilaton background, and point out the loop-hole mentioned above.

The new feature of the dilaton background is that, while 25 of the discrete states correspond to translation symmetry preserved by the background, surprisingly an additional discrete state is present, without an apparent spacetime symmetry corresponding to it. The question is: is there a less obvious spacetime symmetry corresponding to this discrete state, or is this discrete state an exception in the special role played by all other discrete states?

To answer this question, we compute correlation functions involving this discrete state and make the observation in section 4 that this state does not decouple from other physical states, although it is a zero-norm state. ${ }^{1}$ This suggests that the symmetry generated by this state is broken, if there is really a (hidden) symmetry behind it. But we also make the observation that there are strong constraints on the kinematics of the theory

$$
\sum_{a} k_{a}+i \chi_{\mathcal{M}} V=0
$$

where $k_{a}$ 's are external momenta, $\chi_{\mathcal{M}}$ is the Euler character of the string worldsheet and $V$ is the gradient of the dilaton field. Notice that these constraints, although exhibiting the non-conservation of energy-momentum, are equally powerful as the $V=0$ case, for which the constraint is equivalent to the existence of translation symmetry.

In section 5, we elucidate the meaning of these constraints in field theory models, and propose that this example is calling for a generalization of our notion of symmetry. Since the constraint (1.1) gives mathematically as much information as translation symmetry,

\footnotetext{
${ }^{1}$ Here by "zero-norm state" we mean a state that has vanishing norm. Usually, "zero-norm state" is a synonym of physical spurious states. But this discrete state is not spurious.
} 
we should extend our notion of symmetry to incorporate deformations of symmetry of this sort. Finally, we make concluding remarks in section 6 .

\section{Discrete states}

The concept of discrete states [8] is one of the most crucial ideas in 2D string theory. The infinitely many discrete states correspond to an infinite dimensional symmetry algebra (the algebra of area-preserving diffeomorphisms) [9, which can be used to uniquely determine all correlation functions in 2D string theory. In this paper, we would like to generalize the notion of discrete states to $26 \mathrm{D}$ bosonic string theory.

The discrete states in 2D string theory have a few salient features:

1. In the formulation of the old covariant quantization, the physical spectrum admits additional physical states at certain special (discrete) values of the momentum due to a degeneracy of Virasoro constraints or spurious states, in contrast with generic values of momentum.

2. These states indicate a breakdown of the equivalence between the old covariant quantization (or the BRST quantization) and the light-cone gauge, which is essentially the no-ghost theorem.

3. They are associated with symmetries of the theory.

In the light-cone gauge, zero-norm and negative-norm states which are potential threats to the unitarity are eliminated by gauge-fixing. This means that, in the old covariant quantization, physical conditions (Virasoro constraints) are strong enough, and gauge transformations (spurious states) are plenty enough to eliminate all the negative-norm states. As mentioned above in the first property of discrete states, the existence of the discrete states is either due to a weakness of the Virasoro constraint or the lack of spurious states at particular momenta. The second property is therefore closely related to the first. The third property is less directly related to the other two, but is the main reason why discrete states plays a very important role in 2D string theory. In the following, we will extend the use of this terminology and refer to a state in any string theory as "discrete state" if the first two properties are satisfied.

\subsection{Discrete states in 2D with space-like linear dilaton background}

For completeness, we briefly review the idea of discrete states in 2D string theory.

Consider the worldsheet action for a bosonic string in the linear dilaton background ${ }^{2}$

$$
S=\frac{1}{4 \pi \alpha^{\prime}} \int_{\mathcal{M}} d^{2} \sigma \sqrt{g}\left(g^{a b} \partial_{a} X \cdot \partial_{b} X+\alpha^{\prime} R(\sigma) V \cdot X\right)+\frac{1}{2 \pi} \oint_{\partial \mathcal{M}} d s \kappa(\sigma) V \cdot X,
$$

\footnotetext{
${ }^{2}$ For noncritical strings, such as $2 \mathrm{D}$ strings, one needs to add the Liouville potential $\mu e^{\alpha X}$ to the Lagrangian, but in those cases we will consider the spectrum of strings in the region far away from the Liouville wall, where the Liouville potential can be ignored. Hence the potential is irrelevant to our consideration.
} 
where $R(\sigma)$ is the worldsheet curvature and $\kappa(\sigma)$, the geodesic curvature of the boundary. This is a conformal field theory (CFT) with energy-momentum tensor

$$
T(z)=-\frac{1}{\alpha^{\prime}}: \partial X^{\mu} \partial X_{\mu}:+V_{\mu} \partial^{2} X^{\mu},
$$

and central charge

$$
c=d+6 \alpha^{\prime} V_{\mu} V^{\mu} .
$$

Since the linear dilaton background leaves the ghost action intact, the ghost central charge remains to be -26 . Demanding that the total central charge vanishes to give an anomalyfree theory, we have the following condition on space-time dimension

$$
d=26-6 \alpha^{\prime} V \cdot V
$$

In particular, if $V=\left(0, \frac{2}{\sqrt{\alpha^{\prime}}}\right)$, we obtain a $2 \mathrm{D}$ string theory in space-like linear dilaton background. It is easy to see in the light-cone gauge that there can be no physical polarizations in $2 \mathrm{D}$ for generic momentum. For example, consider the first excited state $\zeta \cdot \partial X e^{i k \cdot X}$ for an open string (normal ordering is always assumed). The Virasoro conditions read

$$
\begin{aligned}
\left.\left(L_{0}-1\right) \mid \text { phys }\right\rangle & =0 \Rightarrow k \cdot(k+i V)=0, \\
\left.L_{1} \mid \text { phys }\right\rangle & =0 \Rightarrow \zeta \cdot(k+i V)=0,
\end{aligned}
$$

and a gauge transformation is implied by a spurious state

$$
L_{-1}|\psi\rangle=\text { null } \Rightarrow \zeta^{\mu} \sim \zeta^{\mu}+\lambda k^{\mu} .
$$

One can see that for a generic 2-vector $k^{\mu}$, the constraints above have no solutions for the polarization vector $\zeta$. However, when $k^{\mu}=-i V^{\mu}$, the physical conditions (2.5) is trivially satisfied. On the other hand, when $k^{\mu}=0$, the spurious state (2.7) does not exist. Thus we obtain two discrete states at the specific momenta $k^{\mu}=-i V^{\mu}$ and $k^{\mu}=0$. A short analysis shows that in these cases one can choose the material gauge $\zeta^{1}=0$. In fact, it can be shown that the material gauge works at all massive levels. All we need to do in order to find discrete states is looking for primary fields in the Fock space of $X^{0}$. It turns out that there are infinitely many discrete states corresponding to an infinite dimensional symmetry of $2 \mathrm{D}$ strings.

\subsection{Discrete states in 26D flat space}

It may appear to some readers that the existence of the discrete states rely on the choice of a very special background. But we will show here that there are discrete states even for the background of flat spacetime.

For the 26D flat background, a generic state at the massless level $\left(k^{2}=0\right)$ is of the form

$$
|\psi\rangle=\zeta \cdot \alpha_{-1}|0 ; k\rangle
$$

For zero momentum $k=0$, the Virasoro constraint

$$
L_{1}|\psi\rangle=0
$$


is satisfied for any polarization $\zeta^{\mu},(\mu=0,1, \cdots 25)$. Furthermore, there is no spurious state with zero momentum, since

$$
L_{-1}|0 ; 0\rangle=0 .
$$

Therefore, we get an enlarged spectrum of physical states at $k=0$ : there are 26, instead of 24, physical polarizations of the massless vector field at $k=0$. We will refer to these states as discrete states, because they share the same features as the discrete states in 2D.

This may seem a bit weird, since we have the no-ghost theorem asserting that the spectrum of old covariant quantization is isomorphic to the spectrum in the light-cone gauge, which has only 24 physical polarizations. How can there be a mismatch at discrete states? Apparently, there is a crack in the proof of the no-ghost theorem. We will show where the crack is in section 3.2. More importantly, is unitarity lost due to discrete states? All the discrete states with space-like polarizations have positive norm, but the one with a time-like polarization has negative norm. (One can also superpose them to obtain a state with a light-like polarization, which has zero norm.) Hence there is a "ghost" in the spectrum. Unitarity would be violated if the ghost can be generated in a scattering process. Luckily, the probability of generating a discrete state is zero, because the phase space available for the discrete state has measure zero. Therefore the existence of discrete states does not imply the violation of unitarity, regardless of whether they have positive or negative norms.

If discrete states cannot be generated in scattering, does this mean that they are meaningless in string theory? Like discrete states in 2D, discrete states in 26D flat spacetime are also generators of spacetime symmetry. The vertex operators corresponding to the discrete states $\alpha_{-1}^{\mu}|0 ; 0\rangle$ are $\partial X^{\mu}$, which are the conjugate momenta of $X^{\mu}$. Thus the discrete states at $k=0$ have a clear physical meaning: they generate the spacetime translation symmetry. All the 26 discrete states must therefore decouple from all physical states, as a statement of momentum conservation.

\section{Discrete states in light-like linear dilaton background}

In the previous section we learned two things. First, discrete states exist not only in $2 \mathrm{D}$, but also in 26D. Second, discrete states seem to always generate symmetries. In the following we will test these two observations in the light-like linear dilaton background.

The worldsheet action of a bosonic string in the linear dilaton background was given in (2.1). From (2.4), it is easy to see that if we have a light-like linear dilaton background $(V \cdot V=0)$, we get a $26 \mathrm{D}$ critical string theory. Let us review the basic ingredients of this CFT. Taking the Laurent expansion of (2.2),

$$
T(z)=\sum_{m=-\infty}^{\infty} \frac{L_{m}}{z^{m+2}}, \quad L_{m}=\oint d z z^{m+1} T(z),
$$

one obtains the Virasoro generators

$$
L_{m}=\frac{1}{2} \sum_{n=-\infty}^{\infty} \alpha_{m-n}^{\mu} \alpha_{\mu n}+i \sqrt{\frac{\alpha^{\prime}}{2}}(m+1) V^{\mu} \alpha_{\mu m} .
$$


The OPE of $X, T$, and $L_{n}$ are independent of the linear dilaton background, because the linear dilaton background is a topological effect. It does not change the field equations and we have the same mode expansion

$$
\partial X^{\mu}(z)=-i\left(\frac{\alpha^{\prime}}{2}\right)^{1 / 2} \sum_{m=-\infty}^{\infty} \frac{\alpha_{m}^{\mu}}{z^{m+1}},
$$

and the same canonical commutation relations

$$
\left[\alpha_{m}^{\mu}, \alpha_{n}^{\nu}\right]=m \delta_{m+n} \eta^{\mu \nu}
$$

as in flat spacetime. (Here $p^{\mu}=\left(\frac{2}{\alpha^{\prime}}\right)^{1 / 2} \alpha_{0}^{\mu}$.) One can see this also by separating $X^{\mu}(\sigma)$ into the homogeneous solution $X_{h}^{\mu}(\sigma)$ and the special solution $X_{s}^{\mu}(\sigma)$ (see the appendix). $X_{h}^{\mu}(\sigma)$ has the same field equations and boundary conditions as in flat spacetime, and hence the same OPE. Using conformal transformations, we can set $X_{s}^{\mu}(\sigma)=0$ everywhere except a point with curvature singularity, which can be pushed to infinity such that $X_{s}^{\mu}(\sigma)$ does not affect the OPE.

\subsection{Discrete states at massless level}

To find discrete states in this theory, we take a closer look at the physical conditions and spurious states for the light-like linear dilaton background. The Virasoro constraints are

$$
\left(L_{n}-\delta_{n, 0}\right)|\mathrm{phys}\rangle=0, \quad \forall n \geq 0 .
$$

We will use the convention that $\alpha^{\prime}=\frac{1}{2}$ for the open string theory.

The spurious states are those orthogonal to all physical states. In flat spacetime, the spurious states are $\left\{L_{-m}|\chi\rangle\right\}$, where $m>0$ and $|\chi\rangle$ is an arbitrary state. Since $L_{m}^{\dagger}=L_{-m}$, the inner product of a spurious state with any physical state $|\psi\rangle$ is

$$
\left\langle\psi\left|L_{-m}\right| \chi\right\rangle=\left(L_{m}|\psi\rangle\right)^{\dagger}|\chi\rangle=0
$$

due to Virasoro constraints on the physical state. However, in the linear dilaton background, the adjoint of $L_{-m}$ equals $L_{m}$ with shifted momentum $\hat{p}^{\mu} \rightarrow \hat{p}^{\mu}-i V^{\mu}$. That is, since $\alpha_{\mu m}^{\dagger}=\alpha_{\mu(-m)}$, we have

$$
\begin{aligned}
{\left[L_{-m}(\hat{p})\right]^{\dagger} } & =L_{m}(\hat{p})-2 i \sqrt{\frac{\alpha^{\prime}}{2}} V^{\mu} \alpha_{\mu m} \\
& =\left(\hat{p}^{\mu}-i V^{\mu}\right) \alpha_{\mu m}+\frac{1}{2} \sum_{n \neq 0} \alpha_{m-n} \cdot \alpha_{n}+i \sqrt{\frac{\alpha^{\prime}}{2}}(m+1) V^{\mu} \alpha_{\mu m} \\
& =L_{m}(\hat{p}-i V) .
\end{aligned}
$$

The two-point function of vacuum states in the light-like linear dilaton background is given by

$$
\left\langle 0 ; k \mid 0 ; k^{\prime}\right\rangle=(2 \pi)^{d} \delta\left(k^{\prime}-k+i V\right)
$$


where $\langle 0 ; k|$ and $\left|0 ; k^{\prime}\right\rangle$ are left and right eigenstates of the momentum operator $\hat{p}$

$$
\left\langle 0 ; k\left|\hat{p}=\left\langle 0 ; k|k, \quad \hat{p}| 0 ; k^{\prime}\right\rangle=k^{\prime}\right| 0 ; k^{\prime}\right\rangle .
$$

Since (3.7) should be interpreted as the norm squared $\left.\| 0 ; k^{\prime}\right\rangle\left.\right|^{2}$ when it is non-vanishing, the Hermitian conjugate of a state should have its momentum shifted by $-i V^{\mu}$, i.e.

$$
(|0 ; k\rangle)^{\dagger}=\langle 0 ; k-i V|, \quad \text { or equivalently, } \quad\langle 0 ; k|=(|0 ; k+i V\rangle)^{\dagger} .
$$

This cancels the momentum shift of $L_{-m}^{\dagger}$ exactly. Indeed

$$
\left\langle\psi ; k\left|L_{-m}(\hat{p})\right| \chi ; k^{\prime}\right\rangle=\left\langle\psi ; k\left|\left[L_{m}(\hat{p}-i V)\right]^{\dagger}\right| \chi ; k^{\prime}\right\rangle=\left\{L_{m}(\hat{p}-i V)|\psi ; k+i V\rangle\right\}^{\dagger}\left|\chi ; k^{\prime}\right\rangle=0,
$$

by the physical condition on $|\psi ; k\rangle$. The spurious states are thus still of the same form $\left\{L_{-m}|\chi\rangle\right\}$ as in flat spacetime.

Now we study the physical spectrum at the massless level. A generic first excited state $|\psi\rangle$ can be written as $\zeta \cdot \alpha_{-1}|0 ; k\rangle$, which is subject to the physical conditions

$$
\begin{aligned}
\left(L_{0}-1\right)|\psi\rangle & =0 \Rightarrow k \cdot(k+i V)=0, \\
L_{1}|\psi\rangle & =0 \Rightarrow \zeta \cdot(k+i V)=0 .
\end{aligned}
$$

The only spurious state at this level is

$$
L_{-1}|\psi\rangle=k \cdot \alpha_{-1}|\psi\rangle \Rightarrow \zeta^{\mu} \sim \zeta^{\mu}+\lambda k^{\mu} .
$$

This is formally the same as the $2 \mathrm{D}$ case, but now we have 26 space-time dimensions, and the above equation does have non-trivial solutions for generic momentum $k$. The constraint $\zeta \cdot(k+i V)=0$ reduces one degree of freedom and the gauge symmetry $\zeta^{\mu} \sim \zeta^{\mu}+\lambda k^{\mu}$ eliminates another. As a result, they admit 24 physical polarizations, in agreement with the light-cone gauge.

However, at $k=-i V$ the constraint on polarization becomes trivial and we have one additional physical excitation

$$
\left|D^{-}\right\rangle \equiv \alpha_{-1}^{-}|0 ;-i V\rangle \quad \leftrightarrow \quad \partial X^{-} e^{i(-i V) \cdot X}=\partial X^{-} e^{-V^{-} X^{+}} .
$$

Here we choose the convention that the only nonvanishing component of $V$ is $V^{-}$. Similarly, at $k=0$ there is no spurious state, and the physical spectrum is enlarged. The physical states with $k=0$ are

$$
\begin{aligned}
\left|D^{+}\right\rangle & \equiv \alpha_{-1}^{+}|0 ; 0\rangle \quad \leftrightarrow \quad \partial X^{+}, \\
\left|D^{i}\right\rangle & \equiv \alpha_{-1}^{i}|0 ; 0\rangle \quad \leftrightarrow \quad \partial X^{i}
\end{aligned}
$$

where the index $i$ is used for the 24 transverse directions. We will refer to these states as discrete states for the same reason we used this terminology for the flat spacetime in section 2.2. All these discrete states are one-to-one matched with those in $26 \mathrm{D}$ flat spacetime. Obviously $\left|D^{+}\right\rangle$and $\left|D^{i}\right\rangle$ are still generators of translation symmetries in spacetime. It is unexpected that $\left|D^{-}\right\rangle$is present since the translation symmetry in $X^{+}$is broken by the dilaton background. 


\subsection{No-ghost theorem}

Let us now examine the argument of no-ghost theorem [10] following the presentation in [1], and see how the equivalence between light-cone gauge and BRST quantization breaks down on discrete states. We will skip the proof of the equivalence between the old covariant quantization and BRST quantization, which does not suffer the same problem.

First, we try to prove the no-ghost theorem for the linear dilaton background, by adapting the proof for 26D flat space in [1]. The proof is composed of two parts. The first part of the proof is to find the cohomology of a simplified BRST operator $Q_{1}$, which has the same physical content as the light-cone gauge theory. The second is to show that the cohomology of the full BRST charge $Q_{B}$ is identical to that of $Q_{1}$.

To begin, define the light-cone oscillators

$$
\alpha_{m}^{ \pm}=2^{-1 / 2}\left(\alpha_{m}^{0} \pm \alpha_{m}^{1}\right) .
$$

They satisfy the commutation relation

$$
\left[\alpha_{m}^{+}, \alpha_{n}^{-}\right]=-m \delta_{m+n}, \quad\left[\alpha_{m}^{+}, \alpha_{n}^{+}\right]=\left[\alpha_{m}^{-}, \alpha_{n}^{-}\right]=0 .
$$

We also define the number operator

$$
N^{l c}=\sum_{\substack{m=-\infty \\ m \neq 0}}^{\infty} \frac{1}{m} \alpha_{-m}^{+} \alpha_{m}^{-} .
$$

It counts the number of $X^{-}$excitations minus the number of $X^{+}$excitations.

Now decompose the BRST generator according to the value of $N^{l c}$ as

$$
Q_{B}=Q_{1}+Q_{0}+Q_{-1},
$$

where $Q_{j}$ changes $N^{l c}$ by $j$ units

$$
\left[N^{l c}, Q_{j}\right]=j Q_{j}
$$

Expanding $Q_{B}^{2}=0$ gives

$$
\left(Q_{1}^{2}\right)+\left(\left\{Q_{1}, Q_{0}\right\}\right)+\left(\left\{Q_{1}, Q_{-1}\right\}+Q_{0}^{2}\right)+\left(\left\{Q_{0}, Q_{-1}\right\}\right)+\left(Q_{-1}^{2}\right)=0 .
$$

Each group in parentheses has a different $N^{l c}$ number and must vanish separately. In particular, $Q_{1}$ and $Q_{-1}$ are nilpotent, hence each defines a cohomology of its own. They are given by

$$
\begin{aligned}
Q_{1} & =-\sqrt{2 \alpha^{\prime}} k^{+} \sum_{\substack{m=-\infty \\
m \neq 0}}^{\infty} \alpha_{-m}^{-} c_{m}, \\
Q_{-1} & =-\sqrt{2 \alpha^{\prime}} \sum_{\substack{m=-\infty \\
m \neq 0}}^{\infty}\left[k^{-}+\frac{i}{2} V^{-}(1-m)\right] \alpha_{-m}^{+} c_{m} .
\end{aligned}
$$


Assuming that $k^{+} \neq 0$, we introduce the operator

$$
R=\frac{1}{\left(2 \alpha^{\prime}\right)^{1 / 2} k^{+}} \sum_{\substack{m=-\infty \\ m \neq 0}}^{\infty} \alpha_{-m}^{+} b_{m}
$$

such that

$$
\begin{aligned}
S \equiv\left\{Q_{1}, R\right\} & =\sum_{m=1}^{\infty}\left(m b_{-m} c_{m}+m c_{-m} b_{m}-\alpha_{-m}^{+} \alpha_{m}^{-}-\alpha_{-m}^{-} \alpha_{m}^{+}\right) \\
& =\sum_{m=1}^{\infty} m\left(N_{b m}+N_{c m}+N_{m}^{+}+N_{m}^{-}\right) .
\end{aligned}
$$

The normal ordering constant is determined by noting that $Q_{1}$ and $R$ both annihilate the ground state. Because $Q_{1}$ commutes with $S$, we can calculate the $Q_{1}$ cohomology within each eigen-space of $S$, and the full cohomology is the union of the result.

If $|\psi\rangle$ is $Q_{1}$-closed with $S|\psi\rangle=s|\psi\rangle$, then for nonzero $s$

$$
|\psi\rangle=\frac{1}{s}\left\{Q_{1}, R\right\}|\psi\rangle=\frac{1}{s} Q_{1} R|\psi\rangle,
$$

and so $|\psi\rangle$ is actually $Q_{1}$-exact. Therefore, the $Q_{1}$ cohomology can be nontrivial only at $s=0$. Clearly, the operator $Q_{1}$ annihilates all states in $\operatorname{ker}(S)$, so they are all $Q_{1}$-closed and there are no $Q_{1}$-exact states in this space. Therefore, we have

$$
\operatorname{cohomology}\left(Q_{1}\right) \cong \operatorname{ker}(S) .
$$

On the other hand, by the definition of $S$, the $s=0$ states have no longitudinal or ghost excitations - $\operatorname{ker}(S)$ is just the Hilbert space of the light-cone gauge $\mathcal{H}_{l c}$. Therefore the $Q_{1}$-cohomology is $\mathcal{H}_{l c}$. This proves the no-ghost theorem for $Q_{1}$.

To complete the proof, we have to show that the $Q_{1}$-cohomology is isomorphic to the $Q_{B}$-cohomology. To achieve this goal, let's consider the operator

$$
S+U \equiv\left\{Q_{B}, R\right\}
$$

where $U=\left\{Q_{0}+Q_{-1}, R\right\}$ and it lowers $N^{l c}$ by one or two units.

For each state $\left|\psi_{0}\right\rangle$ in $\operatorname{ker}(S),{ }^{3}$ one can construct another state

$$
|\psi\rangle=\left(1-S^{-1} U+S^{-1} U S^{-1} U-\cdots\right)\left|\psi_{0}\right\rangle,
$$

which is annihilated by $S+U$, i.e. $|\psi\rangle \in \operatorname{ker}(S+U)$. Now, consider a $Q_{B}$-closed state $|\psi\rangle$. Following the same arguments as above, with $S+U$ replacing $S$, one sees that

$$
\operatorname{cohomology}\left(Q_{B}\right) \cong \operatorname{ker}(S+U) \cong \operatorname{ker}(S) \cong \operatorname{cohomology}\left(Q_{1}\right) \cong \mathcal{H}_{l c}
$$

and the proof is completed. But all this was based on the assumption that $k^{+} \neq 0$ so that $(3.23)$ is well defined.

\footnotetext{
${ }^{3}$ The factor $S^{-1}$ makes sense because it always acts on states with $N^{l c}<0$.
} 
Incidentally, we remark that for the flat spacetime, the discrete states with $k=0$ can never have $k^{+} \neq 0$ in any Lorentz frame, and thus the proof breaks down.

Since the linear dilaton background breaks the Lorentz symmetry, it might happen that some states with momenta $k$ has $k^{+}=0$ but we cannot use Lorentz transformation to make $k^{+}$nonzero even when $k$ is not identically zero. In such cases the role of $Q_{1}$ can be replaced by $Q_{-1}$, with the corresponding operator $R$ defined as

$$
R=\frac{1}{\sqrt{2 \alpha^{\prime}}} \sum_{m \neq 0} \frac{\alpha_{-m}^{-} b_{m}}{k^{-}+\frac{i}{2} V^{-}(1+m)},
$$

so that

$$
S \equiv\left\{Q_{-1}, R\right\}=\sum_{m=1}^{\infty} m\left(N_{n m}+N_{c m}+N_{m}^{+}+N_{m}^{-}\right) .
$$

The same argument above works as long as $k^{-}+\frac{i}{2}(m+1) V^{-} \neq 0$ for all nonzero integer $m$.

On the other hand, if

$$
k^{+}=0, \quad k^{-}=-\frac{i}{2}(1+m) V^{-},
$$

and all other components of $k$ vanish, the no-ghost theorem breaks down. At the massless level, only oscillation operators with indices $|m| \leq 1$ matters. We discuss each case separately:

1. $m=-1 \Rightarrow k^{\mu}=0$. This corresponds to the operators $\partial X^{\mu}$, corresponding to the states $\left|D^{i}\right\rangle$ and $\left|D^{+}\right\rangle$. In flat space, these states are just the generating currents of translation symmetry we discussed in section 2.2 .

2. $m=1 \Rightarrow k^{\mu}=-i V^{\mu}$. This is just the discrete state $\left|D^{-}\right\rangle$(3.13).

In summary, at the massless level, the equivalence between BRST quantization and light-cone gauge breaks down exactly at discrete states.

\section{Scattering amplitude in light-like linear dilaton background}

In the previous section, we found that there are 26 discrete states in the light-like linear dilaton background. While 25 of them correspond to spacetime translation symmetry, one is tempted to make the conjecture that the state $\left|D^{-}\right\rangle$is also a generator of a certain symmetry. If this is indeed the case, all states of the theory can be organized according to the representations (charge) of the symmetry generated by $\left|D^{-}\right\rangle$. We do not know yet what is the symmetry transformation, but in principle such information can be deduced from the knowledge of correlation functions. For instance, the discrete states should decouple from all states in the trivial representation. In this section, we will try to investigate properties of the group by studying correlation functions. 
For ordinary vertex operators (those which are not discrete states), their correlation functions in the light-like linear dilaton background and those in the flat spacetime are related to each other by a simple formula [3]

$$
S_{\text {dilaton }}^{g, n}=S_{\text {flat }}^{g, n} \cdot C, \quad C=\int_{-\infty}^{\infty} d \tau_{*} e^{-(2 g-2+n) V \cdot p \tau_{*}},
$$

where $S^{g, n}$ is the diagram of genus $g$ for an $n$-point function. This relation can be easily seen from a calculation in the light-cone gauge $X^{+}=p^{+} \tau$. In this gauge, the remaining worldsheet dynamical fields $X^{i}$ are insensitive to the linear dilaton background. The only effect of the dilaton background to the computation of a scattering amplitude is the appearance of an exponential factor $e^{-V \cdot p \tau}$ at each point $\tau$ of the insertion of a joining/splitting operator in a light-cone gauge diagram. A diagram of genus $g$ for an $n$-string scattering process involves the integrals of $(2 g-2+n)$ parameters for the insertion of joining/splitting operators. The overall effect is to multiply the flat space amplitude by the factor $C$ (4.1), where $\tau_{*}$ is the average of insertion points. However, this is only a formal relation since the on-shell conditions are modified by the linear dilaton background, and the scattering amplitude is only defined on-shell. Furthermore, as we have seen in the previous section, the discrete states are missing in the light-cone gauge. The correlation functions of interest to us need to be calculated using the old covariant quantization.

In the appendix, we develop the path integral formulation for the light-like linear dilaton background. The most salient feature of this background is that the energy-momentum conservation law is modified

$$
\sum_{a} k_{a}^{\mu}+i \chi_{\mathcal{M}} V^{\mu}=0
$$

where $\chi_{\mathcal{M}}$ is the worldsheet Euler character. For a Rieman surface with $g$ genus, $b$ boundaries and $c$ cross-caps, the Euler character is

$$
\chi_{\mathcal{M}}=2-2 g-b-c .
$$

We will refer to this constraint (4.2) as the (non-) conservation law of momentum. It is valid for both open and closed strings.

The (non-)conservation law can be easily derived by integrating out the zero modes of $X^{\mu}$ in the path integral. Apparently, there is a one-to-one correspondence between the 26 components of (4.2) and the 26 discrete states.

In the following we will compute open string amplitudes at tree level. The worldsheet is a disk $D_{2}$ and $\chi_{D_{2}}=1$. We will only consider correlation functions composed of tachyons and massless vectors. The time reversal symmetry on the worldsheet implies that a correlation function of an arbitrary number of tachyons and an odd number of massless vectors vanishes identically.

\subsection{Three-point functions}

As a warmup, we start with the simplest case - the three tachyon scattering amplitude. Up to a delta function imposing the momentum (non-)conservation law (4.2), it should 
be a constant that defines the coupling strength for the 3-tachyon interaction. The path integral gives

$$
\begin{aligned}
S_{D_{2}}\left(k_{1} ; k_{2} ; k_{3}\right)= & \left\langle: c\left(y_{1}\right) e^{i k_{1} \cdot X\left(y_{1}\right)}:: c\left(y_{2}\right) e^{i k_{2} \cdot X\left(y_{2}\right)}:: c\left(y_{3}\right) e^{i k_{3} \cdot X\left(y_{3}\right)}:\right\rangle+\text { permutation } \\
=2 i g_{o}^{3} C_{D_{2}}(2 \pi)^{d} \delta^{d} & \left(\sum_{a=1}^{3} k_{a}^{\mu}+i \chi_{D_{2}} V^{\mu}\right) \times \\
& \times\left|y_{12}\right|^{2 \alpha^{\prime} k_{1} \cdot k_{2}+1}\left|y_{23}\right|^{2 \alpha^{\prime} k_{2} \cdot k_{3}+1}\left|y_{31}\right|^{2 \alpha^{\prime} k_{3} \cdot k_{1}+1}
\end{aligned}
$$

The on-shell condition for tachyons asserts that

$$
\frac{1}{\alpha^{\prime}}=k \cdot(k+i V)=\left(k+\frac{i}{2} V\right)^{2}
$$

Using the momentum (non-)conservation law, we can rewrite the quantities $k_{a} \cdot k_{b}$, e.g.

$$
2 \alpha^{\prime} k_{1} \cdot k_{2}=-1+2 i \alpha^{\prime}\left(\chi_{D_{2}}-1\right) V \cdot k_{3}=-1
$$

for the disk diagram. The three tachyon amplitude is thus

$$
i g_{0}^{3} C_{D_{2}}(2 \pi)^{d} \delta^{d}\left(\sum_{a=1}^{3} k_{a}^{\mu}+i V^{\mu}\right) .
$$

Similarly, the correlation function of one tachyon with two massless vectors is

$$
S_{D_{2}}\left(k_{1} ; \xi, k_{2} ; \zeta, k_{3}\right) \sim\left(-\xi \cdot k_{1} \zeta \cdot k_{1}+\xi \cdot \zeta\right)
$$

\subsection{Four-point functions}

Before computing four-point functions, we introduce Mandelstam variables as follows

$$
\begin{aligned}
& s=\alpha^{\prime}\left(k_{3}+k_{4}\right) \cdot\left(k_{1}+k_{2}\right)+1, \\
& t=\alpha^{\prime}\left(k_{2}+k_{4}\right) \cdot\left(k_{1}+k_{3}\right)+1, \\
& u=\alpha^{\prime}\left(k_{1}+k_{4}\right) \cdot\left(k_{2}+k_{3}\right)+1 .
\end{aligned}
$$

Note that due to the modification of momentum conservation, the generalization of the original Mandelstam variables to the linear dilaton background is ambiguous. In flat space, it is equivalent to write $s=-\alpha^{\prime}\left(k_{1}+k_{2}\right)^{2}$ or $s=-\alpha^{\prime}\left(k_{3}+k_{4}\right)^{2}$. But since we have $k_{1}+k_{2}+k_{3}+k_{4}=-i V$ in the linear dilaton background, these two expressions are not the same.

We find the definitions above convenient. They transform simply under the exchange of two momenta. For example, under the exchange $\left(k_{1} \leftrightarrow k_{2}\right)$, they transform as

$$
s \leftrightarrow s, \quad t \leftrightarrow u, \quad u \leftrightarrow t
$$




\subsubsection{Two tachyons and two massless vectors}

The correlation function of 2 tachyons and 2 massless vectors is

$$
\begin{aligned}
& S_{D_{2}}^{4}= \\
& \begin{aligned}
g_{o}^{4} e^{-\lambda} \int_{-\infty}^{\infty} d x\left\langle: c(0) e^{i k_{1} \cdot X(0)}:: c(1) e^{i k_{2} \cdot X(1)}:: c(\infty) \xi \cdot \partial X e^{i k_{3} \cdot X(\infty)}:: \zeta \cdot \partial X(x) e^{-i k_{4} \cdot X(x)}:\right\rangle \\
\quad \quad+\text { permutation }
\end{aligned} \\
& =K(2 \pi)^{d} \delta^{d}\left(\sum_{a=1}^{4} k_{a}^{\mu}+i V^{\mu}\right) \times \\
& \quad \times\left(-4 \alpha^{\prime 2}\left[\zeta \cdot k_{1} \xi \cdot k_{2} s t+\zeta \cdot k_{2} \xi \cdot k_{1} s u+\zeta \cdot k_{3} \xi \cdot k_{4} t u\right]+2 \alpha^{\prime} \zeta \cdot \xi t u\right) \\
& \times\left[\frac{\Gamma(-s) \Gamma(-t)}{\Gamma(u+1)}+\frac{\Gamma(-s) \Gamma(-u)}{\Gamma(t+1)}-\frac{\Gamma(-u) \Gamma(-t)}{\Gamma(s+1)}\right]+\text { permutation, }
\end{aligned}
$$

where $K=i g_{o}^{4} C_{D_{2}}$. To derive this formula, we used the relation

$$
s+t+u=\alpha^{\prime} \sum_{i} m_{i}^{2}+3=1
$$

among the Mandelstam variables. According to (4.9)-(4.11) and (4.13), they are

$$
\begin{aligned}
& s=-2 \alpha^{\prime} k_{3} \cdot k_{4}+1=-2 \alpha^{\prime} k_{1} \cdot k_{2}-1, \\
& t=-2 \alpha^{\prime} k_{2} \cdot k_{4}=-2 \alpha^{\prime} k_{1} \cdot k_{3}, \\
& u=-2 \alpha^{\prime} k_{1} \cdot k_{4}=-2 \alpha^{\prime} k_{2} \cdot k_{3} .
\end{aligned}
$$

The permutation in the expression above can be carried out in two ways. The first way is to exchange the positions of two tachyons, which can be done by simply exchanging $k_{1} \leftrightarrow k_{2}$ and $t \leftrightarrow u$, and the net result is to simply duplicate the above formula. On the other hand, we can choose to exchange the positions of one tachyon and one photon. We checked explicitly that these two different prescriptions of permutation give the same final answer, and this also serves as a check of the conformal symmetry of the light-like linear dilaton background.

Next, we want to check the gauge symmetry, which corresponds to the decoupling of null states. This is not a trivial check in that we have modified the definition of bras and kets in order to make sense of null states. We separate a polarization $\zeta^{\mu}$ into the transverse and longitudinal parts

$$
\zeta=\zeta^{\perp}+\zeta^{\|} k_{4}
$$

where $\zeta^{\perp}$ denotes the transverse polarization vector, and $\zeta^{\|}$parametrizes the magnitude of the longitudinal part. Similarly we have $\xi=\xi^{\perp}+\xi^{\|} k_{3}$. Under this separation, we can simplify the formulas using the relation

$$
\zeta \cdot k_{3}=\zeta^{\perp} \cdot k_{3}+\zeta^{\|} k_{4} \cdot k_{3}=\zeta^{\perp} \cdot k_{3}+\frac{\zeta^{\|}}{2 \alpha^{\prime}}(1-s) .
$$


Now, the amplitude reads

$$
\begin{aligned}
S_{D_{2}}^{4} \sim & -4 \alpha^{\prime 2}\left(\zeta^{\perp} \cdot k_{1} \xi^{\perp} \cdot k_{2}\right) s t+2 \alpha^{\prime}\left(\zeta^{\perp} \cdot k_{1} \xi^{\|}+\xi^{\perp} \cdot k_{2} \zeta^{\|}\right) s t u-\zeta^{\|} \xi^{\|} u^{2} s t \\
& -4 \alpha^{\prime 2}\left(\zeta^{\perp} \cdot k_{2} \xi^{\perp} \cdot k_{1}\right) s u+2 \alpha^{\prime}\left(\zeta^{\perp} \cdot k_{2} \xi^{\|}+\xi^{\perp} \cdot k_{1} \zeta^{\|}\right) s t u-\zeta^{\|} \xi^{\|} t^{2} s u \\
& -4 \alpha^{\prime 2}\left(\zeta^{\perp} \cdot k_{3} \xi^{\perp} \cdot k_{4}\right) u t+2 \alpha^{\prime}\left(\zeta^{\perp} \cdot k_{3} \xi^{\|}+\xi^{\perp} \cdot k_{4} \zeta^{\|}\right) s t u-\zeta^{\|} \xi^{\|} s(s-1) t u \\
& +2 \alpha^{\prime} \zeta^{\perp} \cdot \xi^{\perp} u t .
\end{aligned}
$$

The $\xi^{\|}$terms vanish due to the momentum (non-)conservation law (4.2) and the physical condition

$$
\zeta^{\perp} \cdot\left(k_{1}+k_{2}+k_{3}\right)=-\zeta^{\perp} \cdot\left(k_{4}+i V\right)=0 .
$$

Similarly the $\zeta^{\|}$terms also vanish. The $\zeta^{\|} \xi^{\|}$term vanishes because

$$
u^{2} s t+t^{2} s u+s(s-1) t u=s t u[(s+t+u)-1]=0 .
$$

Therefore, the null state $k \cdot \alpha_{-1}|0 ; k\rangle$ indeed decouples from the physical spectrum. The scattering amplitude is thus

$S_{D_{2}}^{4} \sim-4 \alpha^{\prime 2}\left(\zeta^{\perp} \cdot k_{1} \xi^{\perp} \cdot k_{2}\right) s t-4 \alpha^{\prime 2}\left(\zeta^{\perp} \cdot k_{2} \xi^{\perp} \cdot k_{1}\right) s u-4 \alpha^{\prime 2}\left(\zeta^{\perp} \cdot k_{3} \xi^{\perp} \cdot k_{4}\right) u t+2 \alpha^{\prime} \zeta^{\perp} \cdot \xi^{\perp} u t$.

This expression can be applied to several special cases of interest to us.

If $k_{4}=0$, i.e., if the 4 -th particle is one of the 25 discrete states $\left(\left|D^{+}\right\rangle,\left|D^{i}\right\rangle\right)$, we find $t=u=0$, and the amplitude vanishes identically, as a reflection of the translation symmetry in spacetime. On the other hand, if $k_{4}=-i V$ and $\zeta=-\hat{e}^{+}$, i.e., if the last particle is the discrete state $\left|D^{-}\right\rangle$, the amplitude is not identically zero. To check this, it suffices to give an example with $S_{D_{2}}^{4} \neq 0$. Consider the case when

$$
k_{1}=i \lambda W-i(1 / 2-\mu) V+\mathbf{k}, \quad k_{2}=-i \lambda W-i(1 / 2+\mu) V-\mathbf{k},
$$

where $W=-\hat{e}^{-} / V^{-}$, and $\mathbf{k}$ is a vector perpendicular to both $W$ and $V$. Since $k_{4}=-i V$, the modified momentum conservation implies that $k_{3}=-\left(k_{1}+k_{2}\right)=i V$ and hence $s=1$. The ansatz above for the momenta makes sure that the on-shell conditions for all momenta $k_{a}$ are satisfied as long as

$$
\mu=\frac{1}{2 \lambda}\left(\mathbf{k}^{2}-\frac{1}{\alpha^{\prime}}\right)
$$

The $\xi^{-}$component of the polarization $\xi$ of the 3rd particle is a pure gauge, and for the momenta assignment (4.21), the amplitude $S_{D_{2}}^{4}$ is

$$
S_{D_{2}}^{4} \sim 16 \alpha^{\prime 2} V^{-}\left[\xi^{+} \alpha^{\prime 2} \lambda^{2}+i \alpha^{\prime} \lambda \xi \cdot \mathbf{k}\right]
$$

where we used $t=2 \alpha^{\prime} \lambda=-u$. Thus $\left|D^{-}\right\rangle$is not decoupled from the massless vector field.

\subsection{Summary of correlation functions}

To summarize the results of our calculation of correlation functions, we find that the state $\left|D^{-}\right\rangle$is not decoupled from other physical states, unlike all other discrete states $\left|D^{i}\right\rangle$ and 
$\left|D^{+}\right\rangle$. The fact that the correlation functions of $\left|D^{-}\right\rangle$with an arbitrary number of tachyons always vanish is consistent with the possibility that the tachyon is a trivial representation of the symmetry group generated by $\left|D^{-}\right\rangle$, if there is really a symmetry. However, this fact is merely a result of the worldsheet time-reversal symmetry, and thus it might be nothing but a coincidence.

On the other hand, the (non-)conservation law of momentum (4.2) has 26 components. We can match each discrete state $\left|D^{\mu}\right\rangle$ with each component of this (non-)conservation law (with the "anomaly" of the conservation law matched with the non-zero momentum of $\left.\left|D^{-}\right\rangle\right)$. Although the $k^{-}$component of (4.2) implies that momentum is no longer conserved, it also specifies precisely how it is not conserved. Mathematically it gives as much information (it imposes as much constraint on kinematics) as a statement of momentum conservation. In this sense the significance of $\left|D^{-}\right\rangle$is not a bit less than any other discrete state. We will explore this observation further in the next section by studying generic field theory models with the same type of (non-)conservation laws.

\section{Field theory model}

We would like to understand the implications of the (non-)conservation law (4.2) in the context of field theory. To begin, we consider a toy model of $N$ scalar fields with polynomial interactions. The action is of the form

$$
S=\int d^{d} x e^{V \cdot x}\left[\frac{1}{2} \phi_{A}\left(-\square+m_{A}^{2}\right) \phi_{A}+g_{A B C} \phi_{A} \phi_{B} \phi_{C}+\lambda_{A B C D} \phi_{A} \phi_{B} \phi_{C} \phi_{D}+\cdots\right] .
$$

Since we have $V$ being light-like, the kinetic term can also be written as

$$
\int d^{d} x e^{V \cdot x} \frac{1}{2}\left[\partial_{\mu} \phi_{A} \partial^{\mu} \phi_{A}+m_{A}^{2} \phi_{A}^{2}\right]
$$

It is obvious from the Fourier decomposition of $\phi_{A}$ that the effect of the factor $e^{V \cdot X}=$ $e^{-V^{-} X^{+}}$to the interaction vertices is simply to modify the momentum conservation law to

$$
\sum_{a=1}^{n} k_{a}+i V=0
$$

for an $n$-point vertex with incoming momenta $k_{a}$. This is in agreement with the (non)conservation law (4.2) for the linear dilaton background for $\chi_{\mathcal{M}}=1$.

The propagator in Fourier basis is given by

$$
G\left(k, k^{\prime}\right)=\frac{1}{k \cdot k^{\prime}-m^{2}} \delta\left(k+k^{\prime}-i V\right) .
$$

For a generic Feynman diagram with $v$ vertices, $e$ propagators and $f$ loops $^{4}$ (which is a diagram with $v$ vertices, $e$ edges and $f$ faces), we can draw the diagram without line crossing on a closed surface $C$ with Euler character

$$
v-e+f=\chi_{C} \equiv 2-2 g^{\prime}-c^{\prime},
$$

\footnotetext{
${ }^{4}$ Here we draw the diagram on a closed surface so that the number of loops is always one more than the value one usually counts in field theory due to the "outer loop" which closes the surface.
} 
where $g^{\prime}$ is the number of handles and $c^{\prime}$ the number of cross-caps. The action (5.1) tells us to associate a factor of $e^{V \cdot X}$ for each vertex, and a factor of $e^{-V \cdot X}$ for each propagator, so that the momenta of the external legs of a Feynman diagram satisfy the relation

$$
\sum_{a} k_{a}+i(v-e) V=0
$$

The coefficient $(v-e)$ here should be compared with its counterpart $\chi_{\mathcal{M}}$ in the (non)conservation law (4.2) in the linear dilaton background

$$
v-e=\chi_{C}-f=2-2 g^{\prime}-f-c^{\prime}, \quad \chi_{\mathcal{M}}=2-2 g-b-c .
$$

Imagining that $\phi_{A}$ 's represent spacetime fields of open string oscillation modes, and that a Feynman diagram with propagating lines replaced by strips is the string worldsheet, we would identify a loop in the Feynman diagram as a boundary of the worldsheet Riemann surface, and therefore we are led to the identification

$$
b=f, \quad g=g^{\prime}, \quad c=c^{\prime} .
$$

Thus the two (non-)conservation laws are exactly the same! We believe that this toy model captures the main features of the (non-)conservation law (4.2) in the linear dilaton background.

Due to its close relationship with string theory in light-like linear dilaton background, quantum field theory models with actions of the form (5.1) deserve further discussion.

For some special cases, for example, if

$$
S=\int d^{d} x e^{V \cdot x}\left[\frac{1}{2} \phi_{A} \square \phi_{A}+g_{A B C} \phi_{A} \phi_{B} \phi_{C}\right],
$$

it is possible to define a symmetry transformation

$$
\begin{aligned}
x^{i} \rightarrow L x^{i}, \quad & x^{-} \rightarrow L^{2} x^{-}, \quad x^{+} \rightarrow x^{+}+a, \\
\phi_{A} \rightarrow & L^{-2} \phi_{A}, \quad L \equiv e^{-\frac{V \cdot a}{d-6}},
\end{aligned}
$$

such that the action $S$ is invariant. However, for generic interaction terms and masses, it seems impossible to define symmetry transformation under which the action (5.1) is invariant.

Recall that the translation symmetry $x^{\mu} \rightarrow x^{\mu}+a^{\mu}$ is fully encoded in the constraint of momentum conservation

$$
\sum_{a} k_{a}=0
$$

and is equivalent to the requirement that the action is of the form

$$
S=\int d^{d} x \mathcal{L}(\phi, \partial \phi, \cdots),
$$

where $\mathcal{L}$ has no explicit dependence on $x^{\mu}$. Similarly, the (non-)conservation law (5.6) implies that the action is of the form

$$
S=\int d^{d} x e^{V \cdot x} \mathcal{L}(\phi, \partial \phi, \cdots) .
$$


Since the implication of either momentum conservation (5.12) or (non-)conservation (5.6) are equally powerful constraints, it is a little odd that only one of them always comes from a symmetry. This should be taken as a hint that our notion of symmetry should be generalized to accommodate the momentum (non-)conservation law and other similar cases.

It is tempting to make the conjecture that the string field theory action in light-like linear dilaton background is related to that in flat spacetime in the same way (5.14) is related to (5.13), probably up to certain field redefinitions and gauge fixing. It will be very interesting to check this explicitly.

Incidentally, we remark that the factor $e^{V \cdot X}$ of the kinetic term can be removed by a field redefinition

$$
\phi_{A}=e^{-\frac{1}{2} V \cdot X} \psi_{A}
$$

Since

$$
e^{V \cdot X} \partial_{\mu} \phi_{A} \partial^{\mu} \phi_{A}=\partial_{\mu} \psi_{A} \partial^{\mu} \psi_{A}-\frac{V^{-}}{2} \partial^{+}\left(\psi_{A}^{2}\right),
$$

the action (5.1) becomes

$S=\int d^{d} x\left[\frac{1}{2} \psi_{A}\left(\square+m_{A}^{2}\right) \psi_{A}+g_{A B C} e^{-V \cdot X / 2} \psi_{A} \psi_{B} \psi_{C}+\lambda_{A B C D} e^{-V \cdot X} \psi_{A} \psi_{B} \psi_{C} \psi_{D}+\cdots\right]$.

The kinetic term is now canonical, and the $n$-point vertex receives a factor of $e^{(n-2) V \cdot X / 2}$, giving the new modified conservation law

$$
\sum_{a=1}^{n} k_{a}-i \frac{(n-2)}{2} V=0
$$

This is of course just (5.3) with $k_{a} \rightarrow k_{a}-i V / 2$.

An interesting property of this class of models (5.1) is that due to the difference in (non)conservation laws (5.6) for different topologies of Feynman diagrams, the contribution of quantum corrections is separated from the classical tree level amplitude. For example, the correlation function

$$
\left\langle\tilde{\phi}_{A}\left(k_{1}\right) \tilde{\phi}_{B}\left(k_{2}\right) \tilde{\phi}_{C}\left(k_{3}\right)\right\rangle
$$

receives no quantum correction at all if $k_{1}+k_{2}+k_{3}+i V=0$.

\section{Concluding remarks}

In this paper we propose that physical states which we call "discrete states" play special roles in string theory. It is well known that they generate a huge symmetry in 2D. They also generate the translation symmetry in 26D flat spacetime. We found that there are also discrete states in the 26D light-like linear dilaton background. Interestingly, in addition to the discrete states corresponding to the translation symmetry in the transverse directions $\left(\left|D^{i}\right\rangle,\left|D^{+}\right\rangle\right)$, we have a discrete state $\left|D^{-}\right\rangle$which does not seem to have a simple interpretation as a symmetry generator. 
There are a few facts to keep in mind. First, the discrete state $\left|D^{-}\right\rangle$has zero norm. Usually, zero-norm states are also spurious physical states. $\left|D^{-}\right\rangle$is an exception to this general rule. We do not demand discrete states to obey the usual rule that zero-norm states also have to be spurious, or that negative-norm states have to be decoupled. Usually, zeronorm and negative-norm states imply problems with unitarity if they are not decoupled. If they are coupled to other physical states, there can be a non-zero probability to create zero-norm or negative-norm state from the scattering of physical states, and unitarity is broken. However, if the zero-norm or negative-norm state is a discrete state, the volume of the phase space available in a scattering process is zero, so the probability of creating such states in a physical process is zero, and unitarity is not broken. Therefore, unitarity does not imply that zero-norm and negative-norm discrete states must decouple from physical states.

Having said this, we note that all discrete states except $\left|D^{-}\right\rangle$are decoupled from all physical states, even though they are not spurious states. This may seem a little puzzling at first sight. However this is just a result of the fact that all spacetime fields are in the trivial representation of the translation group. The fact that $\left|D^{-}\right\rangle$is a state with zero norm, and the fact that it is algebraically analogous to the discrete states in 2 dimensional string theory, still strongly suggest that it should play a special role in the theory.

The clue of the role played by $\left|D^{-}\right\rangle$lies in the observation that, although the translation symmetry in the $X^{+}$direction is broken by the dilaton background, we still have 26 (non-)conservation laws (4.2) for the external momenta, just like in flat space. The (non-)conservation law is equally powerful in constraining the dynamics of the theory for an arbitrary value of $V$, including $V=0$. In this sense the "symmetry" of the linear dilaton background is as big as the flat spacetime. The vertex operator $\left|D^{-}\right\rangle$corresponding to the momentum non-conservation in the $k^{-}$direction

$$
\sum_{a} k_{a}^{-}+i \chi_{\mathcal{M}} V^{-}=0
$$

is therefore playing the same role as all other discrete states $\left|D^{i}\right\rangle$, and $\left|D^{+}\right\rangle$, which correspond to the conservation law

$$
\sum_{a} k_{a}^{i}=0, \quad \sum_{a} k_{a}^{+}=0 .
$$

String theory is known to have huge hidden symmetries which ensure all the nice properties such as dualities and self-consistency. A clear and explicit understanding of these symmetries is however never in reach except for 2D strings. Perhaps this is because our concept of symmetry is still too primitive. To conclude, we believe it is important to study field theory models with constraints mimicking the effect of symmetries, and then try to generalize the notion of symmetry to incorporate these structures.

\section{Acknowledgments}

We thank Chong-Sun Chu, Kazuyuki Furuuchi, Takeo Inami, Hsien-Chung Kao, Yu Nakayama, Shunsuke Teraguchi, Wen-Yu Wen and Syoji Zeze for valuable discussions. 
The work is supported in part by the National Science Council, and the National Center for Theoretical Sciences, Taiwan, R.O.C.

\section{A. Path integral for the linear dilaton background}

In this appendix we show how to compute the correlation function in the light-like linear dilaton background. A linear dilaton background modifies the correlation function in two ways. First, it changes the worldsheet boundary condition in the open string case. Second, it modifies the momentum conservation law. The worldsheet action for an open string in the linear dilaton background is given by

$S=\frac{1}{4 \pi \alpha^{\prime}} \int_{\mathcal{M}} d^{2} \sigma \sqrt{g} g^{a b} \partial_{a} X \cdot \partial_{b} X+\frac{1}{4 \pi} \int_{\mathcal{M}} d^{2} \sigma \sqrt{g} R(\sigma) V \cdot X(\sigma)+\frac{1}{2 \pi} \int_{\partial \mathcal{M}} d s \kappa(\sigma) V \cdot X(\sigma)$,

and its variation is

$$
\delta S=-\frac{1}{2 \pi \alpha^{\prime}} \int_{\mathcal{M}} d^{2} \sigma \sqrt{g}\left(\nabla^{2} X-\frac{\alpha^{\prime}}{2} R(\sigma) V\right) \cdot \delta X-\frac{1}{2 \pi \alpha^{\prime}} \int_{\partial \mathcal{M}} d s\left(\partial_{n} X-\alpha^{\prime} \kappa(\sigma) V\right) \cdot \delta X
$$

where $R(\sigma)(\kappa(\sigma))$ is the worldsheet curvature (geodesic curvature of the boundary), and $\partial_{n}$ denotes the normal derivative along the boundary. From this expression, we can read off the equation of motion

$$
\nabla^{2} X^{\mu}(\sigma)=\frac{\alpha^{\prime}}{2} R(\sigma) V^{\mu}, \quad \sigma \in \mathcal{M}
$$

and boundary condition

$$
\partial_{n} X^{\mu}(\sigma)=\alpha^{\prime} \kappa(\sigma) V^{\mu}, \quad \sigma \in \partial \mathcal{M}
$$

for an open string. The Stoke's theorem implies

$$
\int_{\partial \mathcal{M}} d s \partial_{n} X^{\mu}=\int_{\mathcal{M}} d^{2} \sigma \sqrt{g} \nabla^{2} X^{\mu}
$$

so that

$$
\int_{\partial \mathcal{M}} d s \kappa(\sigma)=\frac{1}{2} \int_{\mathcal{M}} d^{2} \sigma \sqrt{g} R(\sigma)
$$

On the other hand, the Gauss-Bonnet theorem says

$$
\frac{1}{2 \pi} \int_{\partial \mathcal{M}} d s \kappa(\sigma)+\frac{1}{4 \pi} \int_{\mathcal{M}} d^{2} \sigma \sqrt{g} R(\sigma)=\chi_{\mathcal{M}}
$$

where $\chi_{\mathcal{M}}$ is the Euler character of the worldsheet (4.3). These two equations (A.6), A.7) give

$$
\frac{1}{2 \pi} \int_{\partial \mathcal{M}} d s \kappa(\sigma)=\frac{1}{4 \pi} \int_{\mathcal{M}} d^{2} \sigma \sqrt{g} R(\sigma)=\frac{\chi_{\mathcal{M}}}{2} .
$$

Using worldsheet conformal symmetry, one can set both $R(\sigma)$ and $\kappa(\sigma)$ constant

$$
\kappa(\sigma)=\frac{\pi \chi_{\mathcal{M}}}{1}, \quad R(\sigma)=\frac{2 \pi \chi_{\mathcal{M}}}{\mathcal{A}}
$$


where $\mathrm{l}$ and $\mathcal{A}$ are the boundary length and worldsheet area, respectively.

Separating the solution $X^{\mu}(\sigma)$ into a special solution and a homogeneous solution, we find the equation of motion and boundary condition simplified as

$$
\begin{array}{lll}
\nabla^{2} X_{s}^{\mu}(\sigma)=\frac{\alpha^{\prime} \pi}{\mathcal{A}} \chi_{\mathcal{M}} V^{\mu}, & \nabla^{2} X_{h}^{\mu}(\sigma)=0, & (\sigma \in \mathcal{M}), \\
\partial_{n} X_{s}^{\mu}(\sigma)=\frac{\alpha^{\prime} \pi}{1} \chi_{\mathcal{M}} V^{\mu}, & \partial_{n} X_{h}^{\mu}(\sigma)=0, & (\sigma \in \partial \mathcal{M}) .
\end{array}
$$

The homogeneous solution satisfies exactly the same set of equations as in the flat space. Therefore, the Green's function in linear dilaton background is

$$
G^{\prime}\left(\sigma_{1}, \sigma_{2}\right)=-\frac{\alpha^{\prime}}{2} \ln \left|z_{1}-z_{2}\right|^{2}-\frac{\alpha^{\prime}}{2} \ln \left|z_{1}-\bar{z}_{2}\right|^{2}+f\left(z_{1}, \bar{z}_{1}\right)+f\left(z_{2}, \bar{z}_{2}\right),
$$

where

$$
f(z, \bar{z})=\frac{\alpha^{\prime} \chi_{\mathcal{M}} V^{\mu}}{8 \mathcal{A}} \int_{\mathcal{M}} d^{2} z^{\prime} e^{2 \omega\left(z^{\prime}, \bar{z}^{\prime}\right)} \ln \left|z-z^{\prime}\right|^{2}+\text { constant }
$$

comes from the special solution, and the constant is determined by requiring that $G^{\prime}$ is orthogonal to the zero mode of $X^{\mu}(\sigma)$.

Now we are ready to derive the correlation function in the linear dilaton background. The generating functional is

$$
\begin{aligned}
Z[J]= & \left\langle\exp \left(i \int d^{2} \sigma J(\sigma) \cdot X(\sigma)\right)\right\rangle \\
= & \int D X \exp \left\{\int_{\mathcal{M}} d^{2} \sigma\left[\sqrt{g}\left(X^{\mu} \frac{\nabla^{2}}{4 \pi \alpha^{\prime}} X_{\mu}-\frac{R(\sigma)}{4 \pi} V \cdot X\right)+i J(\sigma) \cdot X(\sigma)\right]\right\} \\
& \times \exp \left\{\frac{1}{4 \pi \alpha^{\prime}} \int_{\partial \mathcal{M}} d s X \cdot\left[\partial_{n} X-2 \alpha^{\prime} \kappa(\sigma) V\right]\right\} .
\end{aligned}
$$

Expanding $X^{\mu}(\sigma)$ in terms of a complete basis $X_{I}(\sigma)$, we have

$$
\begin{array}{ccc}
X^{\mu}(\sigma)=\sum_{I} x_{I}^{\mu} X_{I}(\sigma), & \nabla^{2} X_{I}=-\omega_{I}^{2} X_{I}, & \left.\partial_{n} X_{I}\right|_{\partial \mathcal{M}}=0, \\
\int_{\mathcal{M}} \delta^{2} \sigma g^{1 / 2} X_{I} X_{J}=\delta_{I J}, & X_{0}=\left(\int_{\mathcal{M}} d^{2} \sigma g^{1 / 2}\right)^{-1 / 2}=\frac{1}{\sqrt{\mathcal{A}}} .
\end{array}
$$

Then

$$
\begin{aligned}
-\frac{1}{4 \pi} \int_{\mathcal{M}} d^{2} \sigma \sqrt{g} R(\sigma) V \cdot X(\sigma) & =-\frac{\chi_{\mathcal{M}}}{2 \sqrt{\mathcal{A}}} V_{\mu} x_{0}^{\mu}-\sum_{I \neq 0} V_{\mu} x_{I}^{\mu} R_{I}, \\
-\frac{1}{2 \pi} \int_{\partial \mathcal{M}} d s \kappa(\sigma) V \cdot X(\sigma) & =-\frac{\chi_{\mathcal{M}}}{2 \sqrt{\mathcal{A}}} V_{\mu} x_{0}^{\mu}-\sum_{I \neq 0} V_{\mu} x_{I}^{\mu} \kappa_{I}, \\
i \int_{\mathcal{M}} d^{2} \sigma J(\sigma) \cdot X(\sigma) & =i J_{0} \cdot x_{0}+i \sum_{I \neq 0} J_{I} \cdot x_{I},
\end{aligned}
$$


where

$$
\begin{aligned}
R_{I} & =\int_{\mathcal{M}} d^{2} \sigma \sqrt{g} \frac{R(\sigma)}{4 \pi} X_{I}(\sigma), \\
\kappa_{I} & =\int_{\partial \mathcal{M}} d s \frac{\kappa(\sigma)}{2 \pi} X_{I}(\sigma), \\
J_{I}^{\mu} & =\int_{\mathcal{M}} d^{2} \sigma J^{\mu}(\sigma) X_{I}(\sigma) .
\end{aligned}
$$

Using the worldsheet conformal symmetry, we have distributed the curvature uniformly on the world sheet, i.e. $R(\sigma)=R=$ constant and $\kappa(\sigma)=\kappa=$ constant. This greatly simplifies the calculation since now we have $R_{I}=\kappa_{I}=0$ from the orthogonality condition of $X_{I}(\sigma)$. It is straightforward to evaluate the zero mode integral as

$$
\prod_{\mu=0}^{d-1} \int d x_{0}^{\mu} \exp \left\{i\left(i \frac{\chi_{\mathcal{M}}}{\sqrt{\mathcal{A}}} V+J_{0}\right) \cdot x_{0}\right\}=i(2 \pi)^{d} \mathcal{A}^{d / 2} \delta^{d}\left(\sqrt{\mathcal{A}} J_{0}+i \chi_{\mathcal{M}} V\right),
$$

where the factor $i$ comes from the Wick rotation $x_{0}^{0} \rightarrow-i x_{0}^{d}$.

The non-zero mode integral is easy to handle as well. We have

$$
\begin{aligned}
Z[J]= & (\text { zero mode part }) \\
& \times \prod_{I \neq 0, \mu} \int d x_{I}^{\mu} \exp \left(-\frac{\omega_{I}^{2} x_{I}^{\mu} x_{I \mu}}{4 \pi \alpha^{\prime}}+i x_{I}^{\mu} J_{I \mu}+\frac{1}{4 \pi \alpha^{\prime}} \int_{\partial \mathcal{M}} d s x_{I}^{\mu} x_{J \mu} X_{I} \partial_{n} X_{J}\right) \\
= & i(2 \pi)^{d} \mathcal{A}^{d / 2} \delta^{d}\left(\sqrt{\mathcal{A}} J_{0}+i \chi_{\mathcal{M}} V\right)\left(\operatorname{det}^{\prime} \frac{-\nabla^{2}}{4 \pi^{2} \alpha^{\prime}}\right)^{-d / 2} e^{-\frac{1}{2} \int_{\mathcal{M}} d^{2} \sigma d^{2} \sigma^{\prime} J(\sigma) \cdot J\left(\sigma^{\prime}\right) G^{\prime}\left(\sigma, \sigma^{\prime}\right)},
\end{aligned}
$$

where in the second line we have used the boundary condition $\left.\partial_{n} X_{I}\right|_{\partial \mathcal{M}}=0$ to eliminate the boundary term. The notation $\operatorname{det}^{\prime}$ means the determinant defined without the zero modes.

Consider the path integral with a product of tachyon vertex operators

$$
A_{\mathcal{M}}^{n}(k, \sigma)=\left\langle e^{i k_{1} \cdot X\left(\sigma_{1}\right)} e^{i k_{2} \cdot X\left(\sigma_{2}\right)} \cdots e^{i k_{n} \cdot X\left(\sigma_{n}\right)}\right\rangle_{\mathcal{M}} .
$$

This corresponds to

$$
J^{\mu}(\sigma)=\sum_{a=1}^{n} k_{a}^{\mu} \delta^{2}\left(\sigma-\sigma_{i}\right) .
$$

In particular, $J_{0}^{\mu}=\frac{1}{\sqrt{A}}\left[\sum_{a=1}^{n} k_{a}^{\mu}\right]$.

The amplitude (A.20) then becomes

$$
A_{\mathcal{M}}^{n}=i C_{\mathcal{M}}^{X}(2 \pi)^{d} \delta^{d}\left(\sum_{a} k_{a}^{\mu}+i \chi_{\mathcal{M}} V^{\mu}\right) \exp \left(-\frac{1}{2} \int_{\mathcal{M}} d^{2} \sigma d^{2} \sigma^{\prime} J(\sigma) \cdot J\left(\sigma^{\prime}\right) G^{\prime}\left(\sigma, \sigma^{\prime}\right)\right)
$$

where

$$
C_{\mathcal{M}}^{X}=\mathcal{A}^{d / 2}\left(\operatorname{det}^{\prime} \frac{-\nabla^{2}}{4 \pi^{2} \alpha^{\prime}}\right)_{\mathcal{M}}^{-d / 2}=\text { constant }
$$


More generally,

$$
\begin{aligned}
\left\langle\prod_{a=1}^{n} e^{i k_{a} \cdot X\left(y_{i}\right)} \prod_{b=1}^{p} \partial_{y} X^{\mu_{b}}\left(y_{b}^{\prime}\right)\right\rangle_{D_{2}}= & i C_{\mathcal{M}}^{X}(2 \pi)^{d} \delta^{d}\left(\sum_{a} k_{a}^{\mu}+i \chi_{\mathcal{M}} V^{\mu}\right) \\
& \times \prod_{a, b=1 a<b}^{n}\left|y_{a}-y_{b}\right|^{2 \alpha^{\prime} k_{a} \cdot k_{b}}\left\langle\prod_{b=1}^{p}\left[v^{\mu_{b}}\left(y_{b}^{\prime}\right)+q^{\mu_{b}}\left(y_{b}^{\prime}\right)\right]\right\rangle_{D_{2}}
\end{aligned}
$$

where $v^{\mu}(y)=-2 i \alpha^{\prime} \sum_{a=1}^{n} \frac{k_{a}^{\mu}}{y-y_{b}}$, and $q$ 's are contracted using $-2 \alpha^{\prime}\left(y-y^{\prime}\right)^{-2} \eta^{\mu \nu}$.

Since the linear dilaton background does not affect the ghost action, the calculation for ghost contribution remains the same. We can still fix three open string vertex operators on the boundary and compensate it with the corresponding ghost determinant.

\section{References}

[1] See e.g., J. Polchinski, String theory. Vol. 1: an introduction to the bosonic string, Cambridge University Press, Cambridge U.K. (1998).

[2] B. Craps, S. Sethi and E.P. Verlinde, A matrix big bang, JHEP 10 (2005) 005 hep-th/0506180;

B. Craps, A. Rajaraman and S. Sethi, Effective dynamics of the matrix big bang, Phys. Rev. D 73 (2006) 106005 hep-th/0601062.

[3] M. Li, A class of cosmological matrix models, Phys. Lett. B 626 (2005) 202 hep-th/0506260;

M. Li and W. Song, Shock waves and cosmological matrix models, JHEP 10 (2005) 073 hep-th/0507185;

B. Chen, The time-dependent supersymmetric configurations in M-theory and matrix models, Phys. Lett. B 632 (2006) 393 hep-th/0508191.

[4] T. Ishino, H. Kodama and N. Ohta, Time-dependent solutions with null Killing spinor in M-theory and superstrings, Phys. Lett. B 631 (2005) 68 hep-th/0509173;

T. Ishino and N. Ohta, Matrix string description of cosmic singularities in a class of time-dependent solutions, Phys. Lett. B 638 (2006) 105 hep-th/0603215.

[5] C.-S. Chu and P.-M. Ho, Time-dependent AdS/CFT duality and null singularity, JHEP 04 (2006) 013 hep-th/0602054; Time-dependent AdS/CFT duality. II: holographic reconstruction of bulk metric and possible resolution of singularity, arXiv:0710.2640;

S.R. Das, J. Michelson, K. Narayan and S.P. Trivedi, Time dependent cosmologies and their duals, Phys. Rev. D 74 (2006) 026002 hep-th/0602107]; Cosmologies with null singularities and their gauge theory duals, Phys. Rev. D 75 (2007) 026002 hep-th/0610053;

F.-L. Lin and W.-Y. Wen, Supersymmetric null-like holographic cosmologies, JHEP 05 (2006) 013 hep-th/0602124.

[6] F. David, Conformal field theories coupled to $2 D$ gravity in the conformal gauge, Mod. Phys. Lett. A 3 (1988) 1651;

J. Distler and H. Kawai, Conformal field theory and $2 D$ quantum gravity or who's afraid of Joseph Liouville?, Nucl. Phys. B 321 (1989) 509;

Y. Nakayama, Liouville field theory: a decade after the revolution, Int. J. Mod. Phys. A 19 (2004) 2771 hep-th/0402009. 
[7] O. Aharony and E. Silverstein, Supercritical stability, transitions and (pseudo)tachyons, Phys. Rev. D 75 (2007) 046003 hep-th/0612031;

J. McGreevy, E. Silverstein and D. Starr, New dimensions for wound strings: the modular transformation of geometry to topology, Phys. Rev. D 75 (2007) 044025 hep-th/0612121;

D. Green, A. Lawrence, J. McGreevy, D.R. Morrison and E. Silverstein, Dimensional duality, Phys. Rev. D 76 (2007) 066004 [arXiv:0705.0550.

[8] A.M. Polyakov, Selftuning fields and resonant correlations in $2 D$ gravity, Mod. Phys. Lett. A 6 (1991) 635.

[9] I.R. Klebanov and A.M. Polyakov, Interaction of discrete states in two-dimensional string theory, Mod. Phys. Lett. A 6 (1991) 3273 hep-th/9109032.

[10] P. Goddard and C.B. Thorn, Compatibility of the dual Pomeron with unitarity and the absence of ghosts in the dual resonance model, Phys. Lett. B 40 (1972) 235.

[11] G.W. Moore, Finite in all directions, hep-th/9305139; Symmetries and symmetry breaking in string theory, hep-th/9308052. 